\title{
Isolated Sinonasal Posttransplantation Lymphoproliferative Disorder: A Clinical and Radiographic Invasive Fungal Sinusitis Look-a-Like
}

\author{
Kyle M. Hatten ${ }^{a} \quad$ Laurie A. Loevner ${ }^{a}$ b James N. Palmer ${ }^{a}$ Nithin D. Adappa ${ }^{a}$ \\ Departments of a Otorhinolaryngology: Head and Neck Surgery and b Radiology, Hospital of the University of \\ Pennsylvania, Ray and Ruth Perelman School of Medicine at the University of Pennsylvania, Pennsylvania, Pa., USA
}

\section{Key Words}

Fungal sinusitis · Sinonasal lymphoma $\cdot$ Skull base surgery Sphenoid sinus

\begin{abstract}
Posttransplantation lymphoproliferative disorder (PTLD) is a known complication of solid organ transplantation with chronic immunosuppression. We present a unique case that illustrates PTLD mimicking invasive fungal sinusitis both clinically and radiographically. This report addresses the critical diagnostic evaluation and management of PTLD arising from the paranasal sinuses. $\quad$ Copyright $\odot 2013$ S. Karger AG, Basel
\end{abstract}

\section{Introduction}

Chronic immunosuppressive regimens for solid organ transplantation are essential for the long-term survival of transplanted organs. However, immunosuppressive regimens are associated with many side effects and complications, among the less common but more serious of which is posttransplantation lymphoproliferative disorder (PTLD) [1]. PTLD is a histologically and genetically heterogeneous disorder that can range from $\mathrm{B}$ cell hyperplasia to polymorphic B cell lymphoma. There have been varying reports of the incidence of PTLD after transplantation, with Walker et al. [2] reporting 6.2\% incidence after lung transplants, 5.2\% after kidney-pancreas transplants, $2.0 \%$ with cardiac transplants, and $1.4 \%$ with liver transplantation. In this study, the variable rates of PTLD among solid organ transplants directly correlate to the level of immunosuppression required after transplantation.

PTLD has a strong correlation with Epstein-Barr virus (EBV) infection due to the seroconversion of EBV naïve recipients after transplantation. This seroconversion induces an unregulated B cell proliferation in an immunosuppressed patient, which may lead to the development of PTLD [2].

Head and neck involvement of PTLD is uncommon in adults, although it is frequently encountered in the pediatric population $[3,4]$. Isolated involvement of the sinonasal cavity has only been reported once in the literature [4]. We describe a unique case of PTLD in the sphenoid sinus that highlights the importance of having a high index of suspicion for PTLD in the differential diagnosis for immunosuppressed patients with sinonasal pathology. Distinguishing sinonasal PTLD from invasive fungal si-

\section{KARGER}

Fax +4161306 1234

E-Mail karger@karger.com

www.karger.com
(C) 2013 S. Karger AG, Basel

0301-1569/12/0746-0339\$38.00/0

Accessible online at:

www.karger.com/orl
Dr. Kyle M. Hatten

Departments of Otorhinolaryngology: Head and Neck Surgery

Hospital of the University of Pennsylvania

3400 Spruce Street, Philadelphia, PA 19103 (USA)

E-Mail kyle.hatten@uphs.upenn.edu 
Table 1. Comparison of findings between IFS and PTLD

\begin{tabular}{lllll}
\hline & Onset & Gross findings & Radiographic features & Histology (stain - findings) \\
\hline Acute IFS & Hours to days & Necrotic Tissue & Osseous Necrosis & $\begin{array}{l}\text { Silver stain/PAS - submucosal invasion } \\
\text { of fungal hyphae }\end{array}$ \\
\hline PTLD & Weeks to months & Soft Tissue & Sclerotic changes & $\begin{array}{l}\text { HE with EBV staining - plasmacytic } \\
\text { hyperplasia ranging to immunoblasts }\end{array}$ \\
\hline
\end{tabular}

$\mathrm{HE}=$ Hematoxylin and eosin; PAS = periodic acid Schiff stain.

nusitis (IFS), which presents in a similar patient population, is critical as the treatment of these two entities varies considerably (see table 1).

\section{Case Report}

A 71-year-old man with a history of heart transplantation presented with headaches and left facial numbness in the V2 distribution 2 weeks prior to admission. His medical history was significant for recent left maxillary dental procedure performed 1 month previously. The patient denied any nasal obstruction, facial pain, diplopia or history of fevers. His immunosuppression regimen included azathioprine, tacrolimus and prednisone due to a history of chronic rejection.

Physical examination was noteworthy solely for the patient's left V2 maxillary division numbness that had been stable over the previous 2 weeks. The remainder of the patient's neurologic exam as well as head and neck exam was within normal limits. His white blood cell count was $6,200 / \mu \mathrm{l}$ and absolute neutrophil count

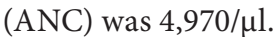

IFS was suspected due to his history of immunosuppression and cranial neuropathy. Magnetic resonance imaging (MRI) showed a central skull base lesion that demonstrated involvement of the left posterior ethmoid air cells and sphenoid sinus with involvement of the left clivus, vidian canal, and the bilateral foramen rotundum (fig. 1).

Preoperative flexible nasal endoscopy showed the patient had intact sensation and no evidence of mucosal pallor or necrosis but, as the patient had no prior endoscopic procedures, we were unable to directly visualize the sphenoid sinus and were concerned that this process may have been ongoing despite a normal endoscopic examination. Endoscopic sinonasal surgery was performed to obtain a tissue diagnosis. There was poor intraoperative visualization of the sphenoid ostium and sinus due to excessive bleeding as the patient was on aspirin for his cardiac transplantation regimen. Postoperative histopathology revealed monomorphic plasmablastic cells consistent with PTLD and did not show any evidence of necrosis or invasion by fungal hyphae. Flow cytometry yielded a clonal B cell lymphoproliferative disorder. In situ hybridization for EBV-encoded RNAs was negative.

The patient's initial management included reduction of his immunosuppressive medication. The azathioprine was discontinued and tacrolimus dosage decreased. Directed radiation therapy was also performed over 12 sessions with a total dosage of $3,960 \mathrm{cGY}$.

\section{Discussion}

IFS classically presents in immunocompromised patients with hematologic malignancies, diabetes mellitus, chronic steroid use and acquired immune deficiency syndrome, as well as solid organ transplantation $[5,6]$. Cranial nerve palsies and radiologic evidence of a sinonasal mass in an immunocompromised individual raises a high index of suspicion for IFS. In such cases, mycotic organisms, particularly mucormycosis and aspergillosis, present with osseous necrosis and invasion of adjacent sinonasal structures often leading to ocular and neurologic symptoms. For IFS, CT has been found to be effective at isolating late-stage boney destruction while MRI is more sensitive at soft-tissue contrast and early-stage findings of extrasinus involvement.

Upon review of the patient's clinical presentation, it is important to note that the patient's 2-week history of facial numbness suggested a diagnosis other than IFS, which is classically an aggressive and rapidly destructive process. Another key reason to search for an alternative diagnosis in this case was the patient's ANC. Parikh et al. [5] reported 45 consecutive cases with IFS that presented with an ANC of less than 1,000, which was not the case in the patient we discuss. It is critical to consider the diagnosis of PTLD in an immunosuppressed patient with an invasive sinonasal mass, particularly in settings without a suppressed ANC.

Cases of PTLD can present at any time and have been reported in transplant recipients as early as 1 month to 10 years after surgery [7]. Patients with head and neck manifestations may present with a mononucleosis-like syndrome, a palpable neck mass, nasal obstruction, as well as facial paresthesia and numbness $[8,9]$. Previous reports of sinonasal PTLD have noted nasal airway obstruction as the primary presenting symptom [8]. Sphenoid sinus and skull base PTLD have rarely been reported [7].

Prompt diagnosis can be obtained with frozen-section biopsy, which can help differentiate PTLD from 

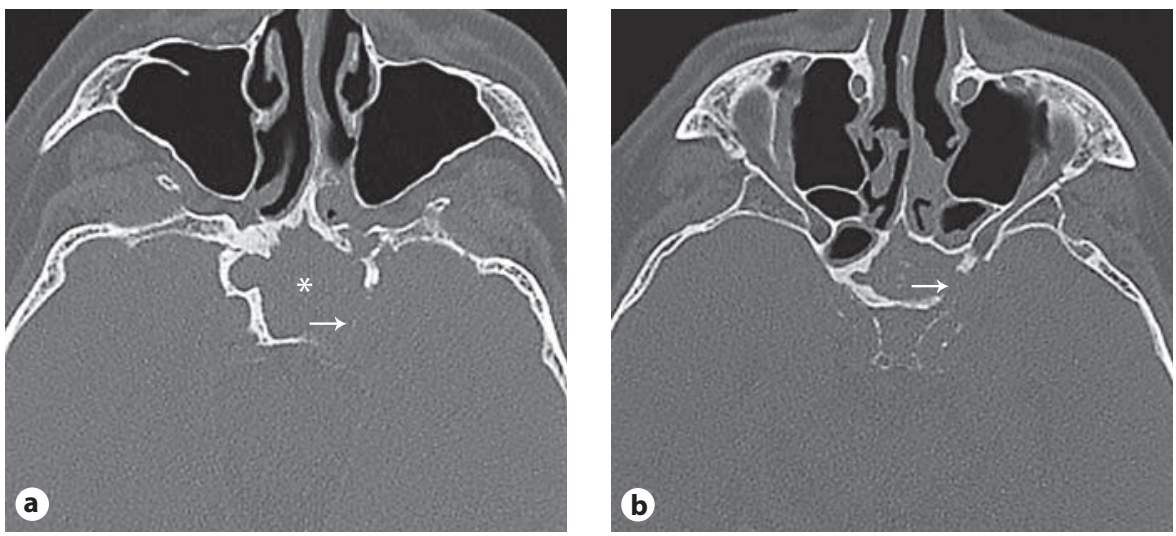

Fig. 1. a, b Axial unenhanced CT scans of the skull base show complete opacification of the sphenoid sinus $\left(^{*}\right)$ with destruction of the left lateral wall of left sphenoid sinus at the level of the internal carotid artery (arrow). c Axial T2-weighted MRI shows hypointense tissue representing lymphoma opacifying the sphenoid sinus and bilateral foramen rotundum $\left(^{*}\right)$. d Sagittal T1-weighted MRI shows lymphoma replacing the sphenoid sinus, involvement of the clivus, and lymphoidal hypertrophy of the adenoids.
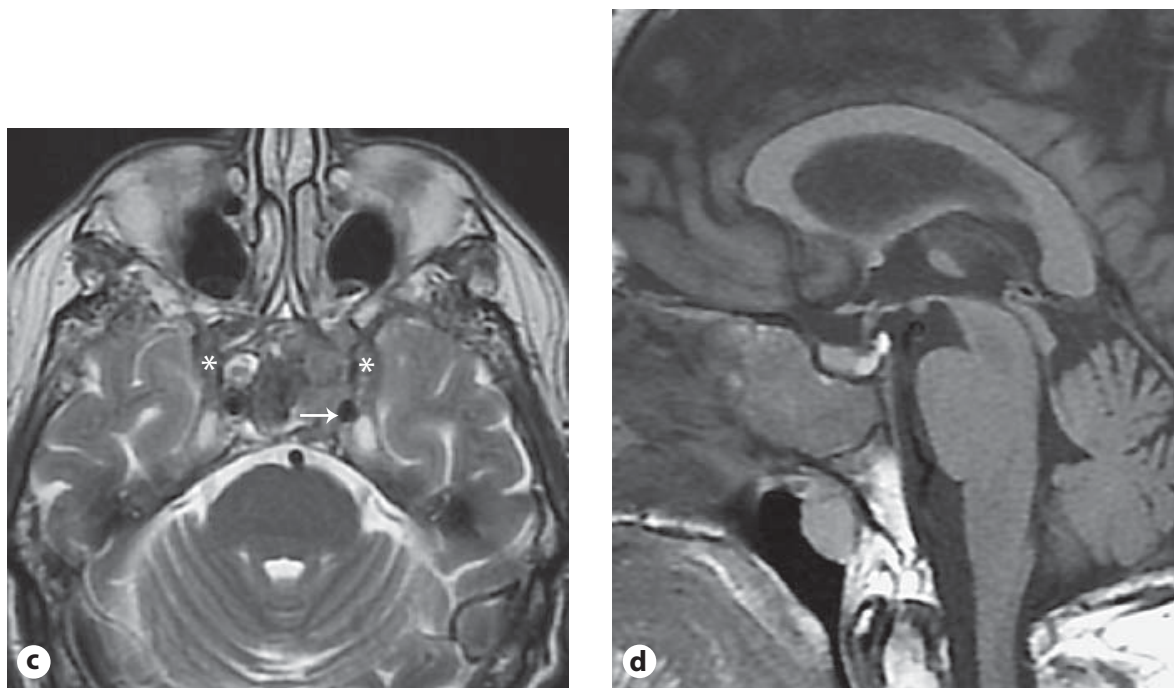

IFS. Frozen-section biopsy for IFS has been noted to have $84 \%$ sensitivity on hematoxylin and eosin staining and assists in establishing the diagnosis as well as planning surgical debridement [10]. Staining for Mucor reveals broad, thin-walled, nonseptate hyphae while Aspergillus is identified by septated, regularly branched hyphae of uniform diameter. IFS histopathologic staining demonstrates coagulative necrosis that is identifiable on hematoxylin and eosin-stained section. Confirmatory stains of angioinvasion by fungal hyphae frequently require special stains with periodic acid Schiff and Grocott staining. PTLD histopathology can vary and may present as small lymphocytes, plasma cells, immunoblasts, or Reed-Sternberg/Hodgkin cells with immunohistochemistry staining (table 1) [11].

Timely diagnosis of PTLD is paramount due to the vastly different treatment compared to IFS. Management of PTLD includes immediate reduction of immunosuppressive therapy [12]. With the advent of immunomodulation and monoclonal antibodies, new therapies of PTLD have been directed towards the use of interferon, intravenous immunoglobulins, anti-B cell antibodies, as well as cellular therapies with cytotoxic T lymphocytes directed against EBV-specific antigens [13]. In certain cases of isolated PTLD, such as in our case, localized radiation therapy may play a pivotal role in the patient's treatment.

\section{Conclusion}

This case illustrates the importance of having a high index of suspicion of PTLD in the differential diagnosis of transplant recipients with aggressive paranasal sinus lesions. Although relatively rare, sinonasal PTLD is probably more common than reported given the ever-increasing population of organ transplantation patients. Prompt diagnosis is critical to initiate the appropriate therapy due to high morbidity and mortality of the respective disease processes and to avoid unnecessary exposure to toxic antifungals that are typically necessary in IFS. 


\section{References}

-1 Fizzera G, Hanto D, Gajl-Peczalska K, et al: Polymorphinc diffuse B-cell hyperplasia and lymphomas in renal transplant recipients. Cancer Res 1981;41:4262-4279.

2 Walker R, Paya C, Marshall W, et al: Pretransplantation seronegative Epstein-Barr virus status is the primary risk factor for posttransplantation lymphoproliferative disorder in adult heart, lung, and other solid organ transplantations J Heart Lung Transplant 1995; 14:214-221.

3 Lattyak B, Rosenthal P, Mudge C, et al: Posttransplant lymphoproliferative disorder presenting in the head and neck. Laryngoscope 1998;108:1195-1198.

4 Gordon AR, Loevner LA, Sonners AI, et al: Posttransplantation lymphoproliferative disorder of the paranasal sinuses mimicking invasive fungal sinusitis: a case report. Am J Neuroradiol 2002;23:855-857.
5 Parikh S, Venkatraman G, DelGaudio J: Invasive fungal sinusitis: a 15 -year review from a single institution. Am J Rhinol 2004;18: 75-81.

6 Gillespie M, O’Malley B, Francis H: An approach to fulminant invasive fungal rhinosinusitis in the immunocomprised host. Arch Otolaryngol Head Neck Surg 1998:124; 520 526.

7 Basgoz N, Preiksaitis JK: Post-transplant lymphoproliferative disorder. Infect Dis Clin North Am 1995;9:901-923.

$\checkmark 8$ Loevner L, Karpati R, Kumar P, et al: Posttransplantation lymphoproliferative disorder of the head and neck: imaging feature in seven adults. Radiology 2000;216:363-369.
9 Herrmann B, Sweet S, Molter D: Sinonasal posttransplant lymphoproliferative disorder in pediatric lung transplant patients. Otolaryngol Head Neck Surg 2005;133:38-41.

10 Ghadiali M, Deckard N, Uzma F, et al: Frozen-section biopsy analysis for acute invasive fungal rhinosinusitis. Otolaryngol Head Neck Surg 2007;136:714-719.

-11 Parker A, Bowles K, Bradley J, et al: Diagnosis of post-transplant lymphoproliferative disorder in solid organ transplant recipients - BCSH and BTS Guidelines. Br J Haematol 2010;149:675-692.

12 Starlz T, Porter K, Rosenthal J, et al: Reversibility of lymphomas and lymphoproliferative lesions developing under cyclosporinsteroid therapy. Lancet 1984;17:583-587.

13 Green M: Management of Epstein-Barr virus-induced post-transplant lymphoproliferative disease in recipients of solid organ transplantation. Am J Transplant 2001;1: 103-118. 